\title{
Using component modes in a system design process
}

\author{
Guillaume Vermot des Roches ${ }^{1}$, Jean-Philippe Bianchi ${ }^{1}$, Etienne Balmes ${ }^{1,2}$, \\ Remi Lemaire ${ }^{3}$, Thierry Pasquet ${ }^{3}$ \\ $1:$ SDTools, 2 : Arts et Metiers ParisTech, \\ 3 : Robert Bosch France (SAS), Division Chassis System Brakes
}

\begin{abstract}
Classically Component Mode Synthesis has been used to couple models from different sources (mixed test and analysis, different companies, different software, ...) or reduce models. The focus of this paper is on using component modes in a design cycle. Component modes are the natural representation in the lowest part design cycle. In NVH applications, it is well known that coupling at the system level makes understanding of the impact of component design changes difficult. The paper details and uses the disjoint component synthesis method. Like classical CMS this synthesis method considers reduced component models and couples these models to obtain a system level synthesis. The first novel aspect is that a physical interface is assumed to exist between components. Synthesis thus becomes a trivial application of model assembly. The second novel aspect is that components are reduced using component modes and the trace of nominal system modes, thus allowing exact predictions for the nominal design. Illustrations of the methodology are given for an automotive brake application.
\end{abstract}

\section{INTRODUCTION}

Numerical prototyping is widely used in industrial design processes to allow optimization and limit the cost of validation through experimental testing. A typical organization of the design is so called V-model shown in figure 1 . In NVH applications, performance can often only be evaluated in the system verification and validation step. It is thus quite difficult to specify requirements or component level verifications.

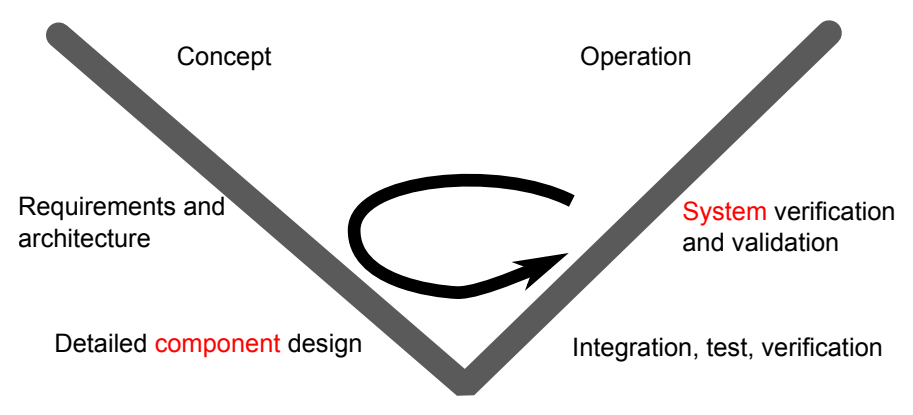

Figure 1: V-model for product development

The purpose of the present work is to introduce a methodology that, given a base system model (current design), leads to a rapid iteration between component level properties and system level predictions. The existence of a base model ensures that the component coupling with its environment is appropriate and will allow system level predictions of performance. The first issue that will be addressed is to build a method that does not require computationally intensive evaluations of performance to validate the impact of component changes. The second issue is to allow the evaluation of design changes in terms of component frequencies and damping rather than detailed model geometry and properties. 
To simplify the integration of components into a system model, section 1.1 introduces the idea of coupling components using physical interfaces rather than the assumption of continuity classically introduced in Component Mode Synthesis (CMS) methods ${ }^{[1,2]}$. With this disjoint component synthesis method, components have fully distinct Degrees Of Freedom (DOF) and one distinguishes component and coupling matrices. Earlier work on this approach can be found in Refs. [3] to cope with incompatible meshes, ${ }^{[4]}$ for structural dynamics modification applications, ${ }^{[5]}$ for damping allocation.

As components are disjoint, their reduction is a direct application of Ritz analysis. The only requirement is to build a basis approximating the component motion. Section 1.2 shows that reduced component models can be built using the trace of system responses thus leading to coupled responses that are exact for the nominal design and very accurate otherwise. In such models, it is not necessary to represent all possible interface deformations as classically done using static interface or constraint modes ${ }^{[1]}$. In a number of cases, this results in much smaller reduced models.

Finally, using disjoint components gives a wide range of options for defining component DOF. Section 1.3 thus discusses how component free/free modes can be used as Rayleigh Ritz vectors. Component mode frequency and damping are then used as design parameters in a specification phase. The models being very small, system performance evaluations are typically very fast.

The use of the proposed methods is then illustrated in section 2 on the case of a state of the art automotive brake model.

\section{CMS PROCEDURES FOR DESIGN}

\subsection{Coupling components with physical interfaces}

Component Mode Synthesis methods ${ }^{[1,2]}$ divide structures into components and generate coupled predictions by introducing assumptions on the interfaces between components. Most of the literature on CMS considers that the fundamental assumption for coupling is the fact that displacement is continuous at interfaces.

The approach, considered here, assumes that the components are physically disjoint and that coupling occurs through interfaces that have a physical extent. Since the components are disjoint, the associated DOF $\left\{q_{c i}\right\}$ are always distinct. Matrices associated with full or reduced components are non zero for a single component, while interfaces can have nonzero values coupling multiple components. Their diagonal blocks are non null on interface component DOF. In the case of two components one thus has

$$
\left[Z_{T}\right]=\left[Z_{e l}\right]+\left[Z_{I}\right]=\left[\begin{array}{cc}
Z_{1} & 0 \\
0 & Z_{2}
\end{array}\right]+\left[\begin{array}{cc}
Z_{I 11} & Z_{I 12} \\
Z_{I 21} & Z_{I 22}
\end{array}\right]
$$

Figure 2 shows the matrix connectivities for a sample 3 component $/ 2$ interface model. The block structure of the component and interface models is indicated through colors. In the application of section 2 the interface matrices correspond to tangent contact/friction coupling models.

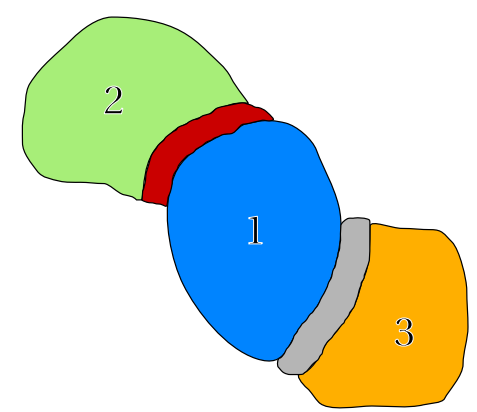

(a) Conceptual model

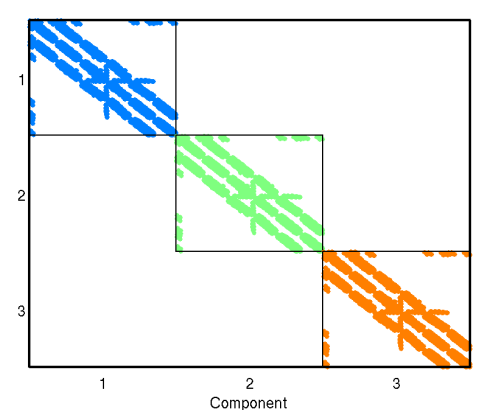

(b) FE elastic matrix

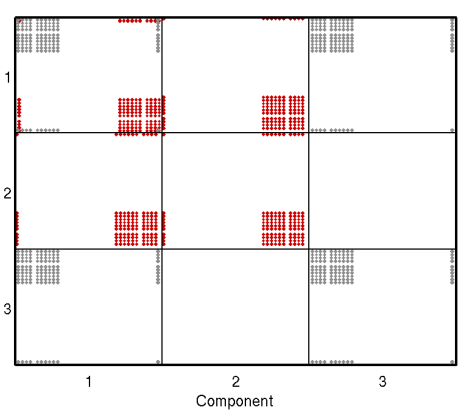

(c) FE interface matrix

Figure 2: Finite element stiffness matrices 
Since DOF associated with components are distinct, model reduction is simply performed by generating an assumed basis (classical Rayleigh Ritz reduction) for each component response

$$
\left\{q_{c i}\right\} \approx\left[T_{c i}\right]\left\{q_{c i R}\right\}
$$

Thus yielding a reduction basis defined on the whole model

$$
\left\{\begin{array}{l}
q_{c 1} \\
q_{c 2}
\end{array}\right\}=\left[\begin{array}{cc}
T_{c 1} & 0 \\
0 & T_{c 2}
\end{array}\right]\left\{\begin{array}{l}
q_{c 1 R} \\
q_{c 2 R}
\end{array}\right\}
$$

Displacements are by nature continuous since they are defined on a single component, and system predictions are readily obtained by reducing the coupling matrices

$$
\left[Z_{I}\right]_{i j R}=\left[T_{c i}\right]^{T}\left[Z_{I}\right]_{i j}\left[T_{c j}\right]
$$

It is important to note that block size for component interface is equal to the number of vectors (2). The number of interface DOF between components thus has no effect on the reduced model size. This feature is a strong deviation from what would be obtained with classical reduction methods, such as the Craig-Bampton method ${ }^{[1]}$, which tend to have very high numerical costs in cases with large interfaces. In the coupling matrix reduction, accounting for block operations on components that are actually coupled often leads to major performance gains.

\subsection{Reduced models with exact modes}

In the proposed setting, computing the nominal model modes is considered to be straightforward. This is nowadays the case using either classical iterative method (Lanczos, Implicitly Restarted Arnoldi, ...) or automated multi-level substructuring methods $[6,7]$. The key point in design procedures is rather the ability to accurately perform parametric studies. It is then critical to compute the system modes several hundred times for reanalysis, which requires the use of reduced models to be performed in reasonable computation times.

In classical CMS methods ${ }^{[1]}$, one assumes component independence : the data from one component should be independent of the data from other components. While this made sense for the purpose of getting faster computations or coupling test and analysis derived models, this assumption is a poor idea in a design cycle. Indeed model precision is altered despite the fact that the nominal design gives appropriate coupling information. It is thus proposed to replace component modes by the trace of the global system modes $[\Phi]$ on each component $c_{i}$

$$
\left[T_{c i}\right]=\left[\Phi_{\mid c i}\right]_{1: N_{s}} \text { Orth }
$$

The clear advantage of this reduction basis is that both reduced and full models share the same modes. Such dynamic behavior equivalence allows to retain a good accuracy for modal based computations like forced response or complex mode computation.

If multiple system configurations are of interest, one can use the trace of multiple mode sets $\left(\left[\Phi_{\mid c i}\left(p_{1}\right) \Phi_{\mid c i}\left(p_{2}\right) \ldots\right]\right)$. Variable rotation speeds are thus considered in ${ }^{[8]}$, where the issue of generating bases from vector sets is also addressed. Fixed basis reduction for variable configurations can be applied to all or specific components.

For compatibility with existing software, it is also possible to include static interface or constraint modes ${ }^{[1]}$. For interfaces with a large number of DOF this is however often very detrimental for the reduced model size. With the present methodology, the component model really only needs as many base vectors as there are independent shapes in the trace of system level modes (for example a very stiff component only needs 6 shapes to describe its rigid body modes). 


\subsection{Using component modes as DOF and design parameters}

In a detailed design phase, component parameters to be optimized are known and the CMS methodology detailed above gives a rapid way to evaluate system level performance. But in a specification phase, one typically seeks to understand how the component behavior impacts system response.

The idea proposed here is to analyze the impact of changes in frequency and damping of the free/free component modes. These represent a series of simple scalar parameters and introducing detailed design changes to shift the frequency or damp specific modes is often a clear process. A similar idea with fixed interface modes is used to study mistuning of bladed disk assemblies in Ref. ${ }^{[8]}$.

To obtain accurate predictions, one now seeks to retain exact modes for both the system and components. For each component $i$, one thus uses a reduction basis that combines component modes in free/free conditions $\left[\phi_{c i}\right]$ and the trace on the component of exact system modes $\left[\Phi_{\mid c i}\right]$

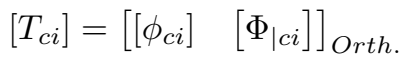

In practice, $\left[\Phi_{\mid c i}\right]$ may not be orthogonal to the component free/free modes, one thus generates an orthonormal basis by some appropriate method ${ }^{[8]}$. The component modes are kept explicitly and are not modified by the orthonormalization process since by definition they are orthonormal to start with. The difference between the subspace of system modes and the component modes is also kept with the only differences linked to numerical round off errors in the orthonormalization process.

Since the component basis is orthonormal, the reduced component mass is identity and the stiffness is diagonal with the first terms corresponding to the square of component mode pulsations. Damping is a full matrix if a damping model exists (non-proportional damping) but in a design phase it is typically assumed to be diagonal (modal damping). Parametric studies on component mode frequencies or modal damping ratio thus simply correspond to changes on the associated diagonal terms of the reduced component models. This process will be illustrated in section 2.2 .

As shown in figure 3 , the interface matrices are now fully populated of blocks corresponding to connected components. When the component basis contains more vectors than interface DOF with another component, specific basis reordering can significantly reduce interface coupling and thus lead to sparse reduced models.

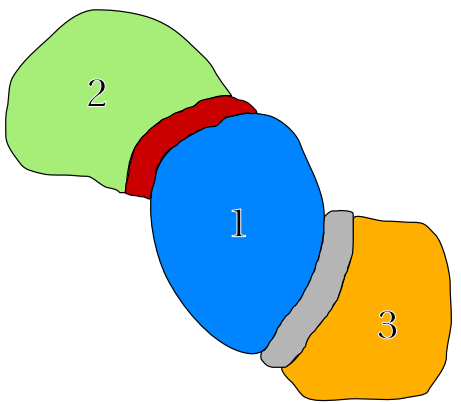

(a) Conceptual model

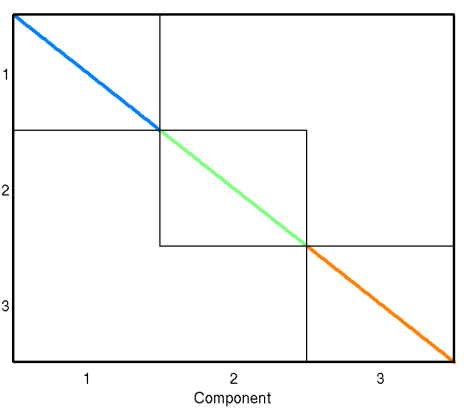

(b) Reduced stiffness matrix

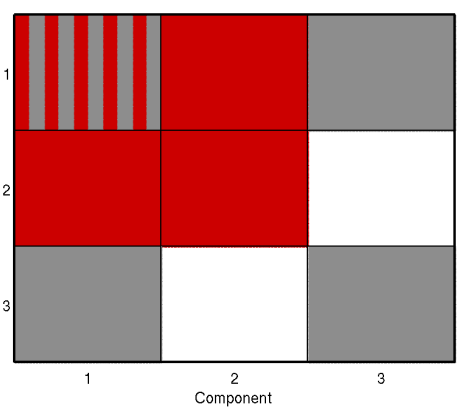

(c) Reduced interface matrix

Figure 3: Reduced stiffness matrices 


\section{APPLICATION TO AN AUTOMOTIVE BRAKE SYSTEM}

\subsection{Test case}

For the illustrations shown in this paper, one considers a state of the art brake model shown in figure 4 The model is an assembly of 8 components in contact with each other finely meshed to comply with the need to compute its modes up to $16 \mathrm{kHz}$ for squeal simulations, thus leading to the use of 600,000 DOF. Static computations of the steady state shape of the brake are performed using ABAQUS. Component and interface models are then imported into SDT ${ }^{[9]}$ whose the superelement utilities are used to manage further computations.
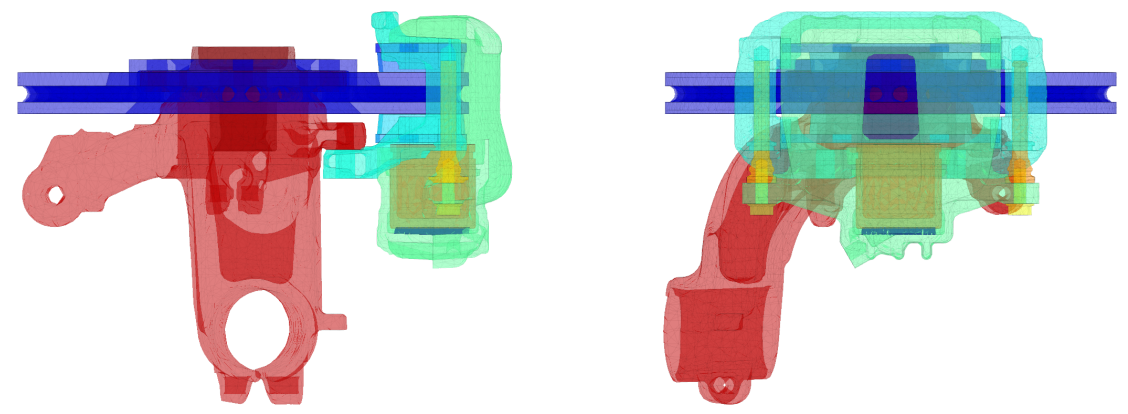

Figure 4: Full brake model

The components are in contact with each other through 11 interfaces representing several thousand DOF. The reduction basis of equation (3) is computed for 8 different components. The reduced model obtained is sparse and contains only 1,300 DOF (for 316 component modes and 250 global modes). With such a small size, only a few seconds are then needed to compute reduced global system modes.

Figure 5 shows the relative frequency error between reduced and full system real modes. The error is within numerical precision for the whole range.

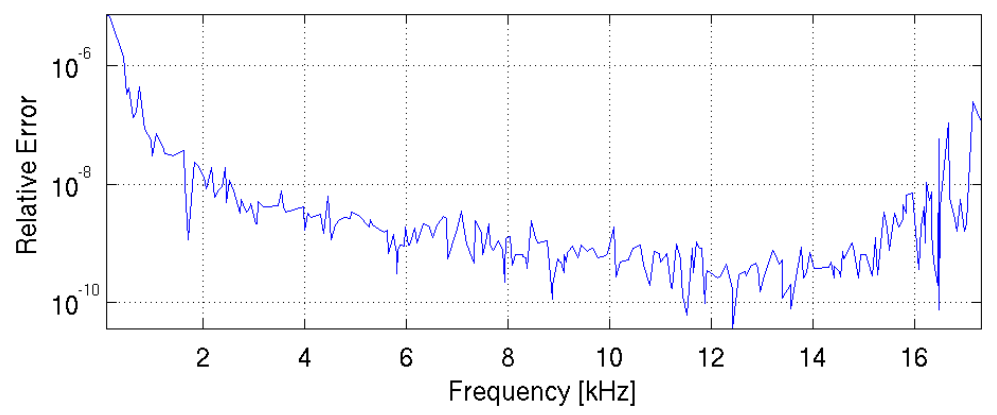

Figure 5: Reduced real mode relative frequency error to full modes

The non-linearities induced by contact and friction yield system instabilities responsible in particular for brake noises (groan, moan, squeal, ...). These instabilities are non destructive and an end goal of the present work is time simulation of the full brake assembly (see ${ }^{[10]}$ for details).

This paper focuses on an earlier part of the design cycle, where performance is analyzed using unstable complex modes and structural modifications are tested for their impact. Section 2.2 illustrates the effectiveness of the proposed reduced model for reanalysis studies. Section 2.3 shows how component modes can be used to guide design. 


\subsection{Reanalysis in design phase}

In a design phase, the ability to rapidly evaluate the impact of modifications is a key advantage of the proposed method. These modifications can be physical (one will consider a modulus or mass change here) or correspond to component modes (which will be illustrated in the next section). The uses of reanalysis are classically perturbation/sensitivity, uncertainty and model updating analyses. The low cost allows the evaluation for a large number of intermediate values. This makes tracking of poles practical as will be illustrated. The two examples given in this section are chosen to illustrate the robustness of the method for significant changes.

The first example shows the effect of the disc Young modulus on the global system modes. Such shift is trivial to apply to a component with an isotropic material. Since the stiffness matrix is proportional to the modulus, the impact of a modulus change is a simple multiplicative coefficient applied to the block of the reduced elastic matrix of figure 2.

Figure 6 shows the prediction accuracy for the system real modes with a $+10 \%$ shift of the Young modulus. Predicted and exact frequency shifts $\Delta f=\left\|f_{\text {nom }}-f_{\text {mod }}\right\|$ are plotted along with the shift error $\Delta \Delta f=\frac{\Delta f_{\text {pred }}-\Delta f_{\text {compt }} \text {. The error }}{\Delta f_{\text {compt }}}$ on predicted shifts is clearly small on the whole frequency range.

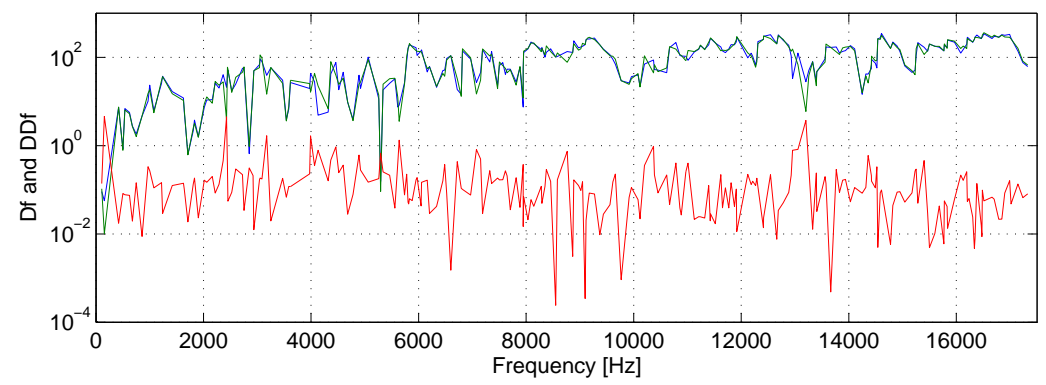

Figure 6: Frequency shift prediction error for a $+10 \%$ disc Young Modulus shift. $-\Delta f_{\text {pred }},-\Delta f_{\text {compt }},-\Delta \Delta f$

Figure 7 shows two complex mode prediction showing high disc participation. It appears that changing the modulus leads to stable/unstable transitions, which is of clear interest in a squeal study. A variation from $-20 \%$ to $+30 \%$ of Young modulus is considered and compared to full order computations at $-20 \%,+10 \%$ and $+20 \%$ modulus shifts. The correspondence is very good and clearly demonstrates the ability to predict complex pole patterns found in stability analysis.
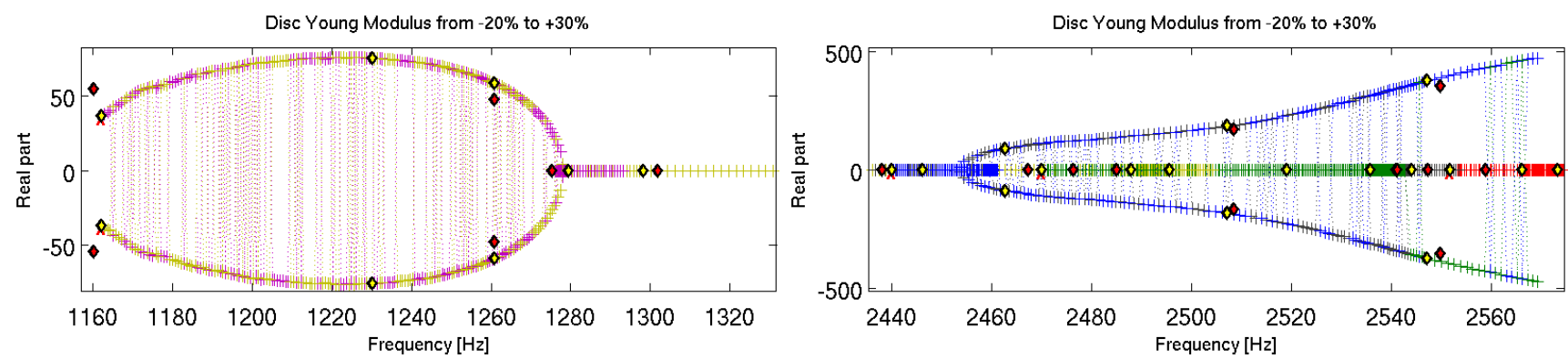

Figure 7: Complex mode evolution prediction for a disc Young modulus variation. red $\diamond$ : full recomputation, yellow $\diamond$ prediction corresponding to recomputed points, green $\diamond$ : nominal point, + : prediction

The second example presents the effect of mass modification on a component. The brake anchor has a so-called handle which drives the anchor rigidity in torsion. The effect of the handle inertia can be studied to set anchor handle modes at frequencies where no interaction involving anchor torsion occurs. The effect of adding a 9 gram point mass at the anchor handle center is considered. 
Figure 8 shows the additional mass effect on all the anchor modes, a variation from nothing to $-6 \%$ is observed. To apply the mass modification, a $\Delta M_{\text {anchor }}$ matrix is computed and projected on the anchor reduction basis which can then be added to the system mass.
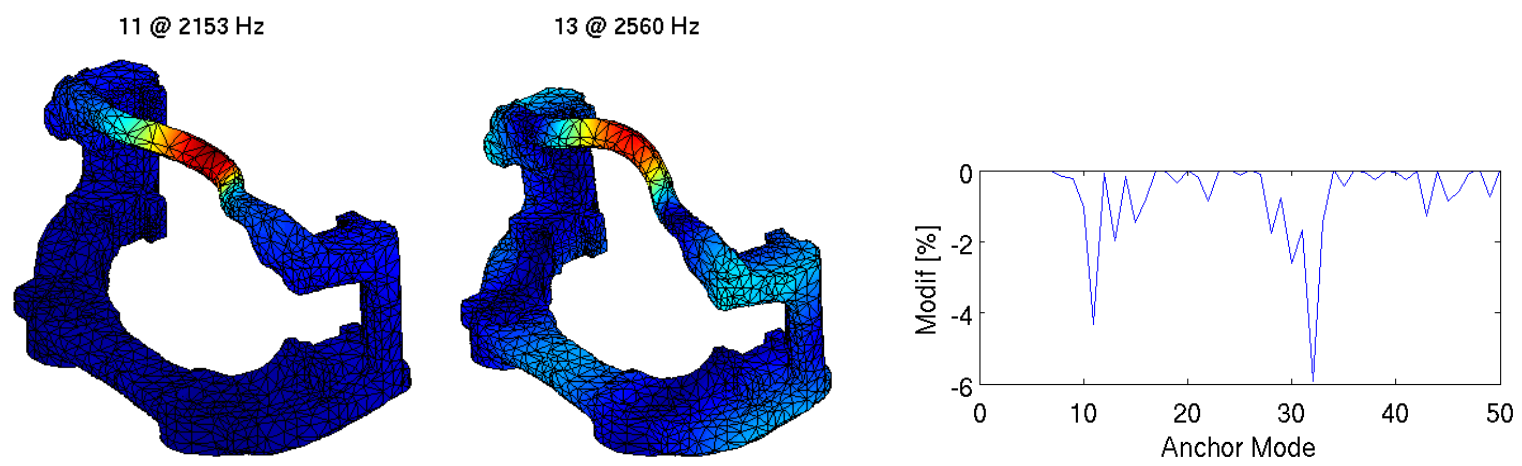

Figure 8: Anchor handle mass modification. Left, original mode \#11 and \#13 (highly impacted by handle design modification). Right, anchor real modes frequency variation

The error on real mode frequencies is presented in figure 9 following the same evaluation than for the disc Young modulus variation. Frequency shifts due to the modification are well predicted. It can be noticed that significant frequency shifts (with high anchor modal participation) show the smallest errors on frequency shift.

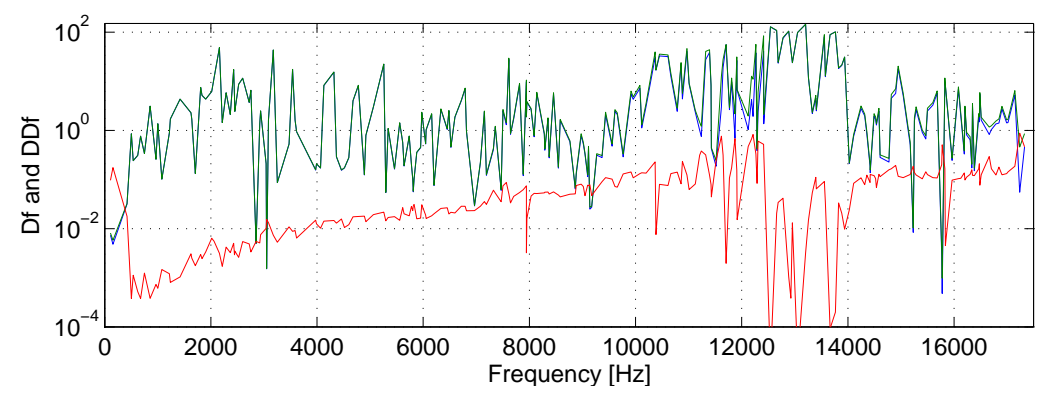

Figure 9: Frequency shift prediction error for a mass variation of the anchor handle. $-\Delta f_{\text {pred }},-\Delta f_{\text {compt }},-\Delta \Delta f$

\subsection{Sensitivity to component modes}

The previous section focused on the structural modification effect predictions. This section focuses on the methodology used to identify components needing modifications. Since component modes appear as explicit DOF in the reduced system matrices, the sensitivity of the stiffness matrix to a frequency change is a matrix with only one non zero element on the diagonal at position $p$. The sensitivity of a complex pole to a change in the $p$ frequency of a component mode is thus simply given by the product of the complex mode components associated with that DOF

$$
\frac{\partial \lambda_{j}}{\partial p}=\left\{\psi_{j L}^{T}\right\}_{p}\left\{\psi_{j}^{T}\right\}_{p}
$$

The sensitivity of all system to all component modes of the reduced model is easily computed in less than 60 seconds. Since the focus is on instabilities, one only considers the damping sensitivity of unstable modes. Figure 10 shows a global sensitivity map. The plot gives a direct illustration of which modes of which component impacts the instabilities. 


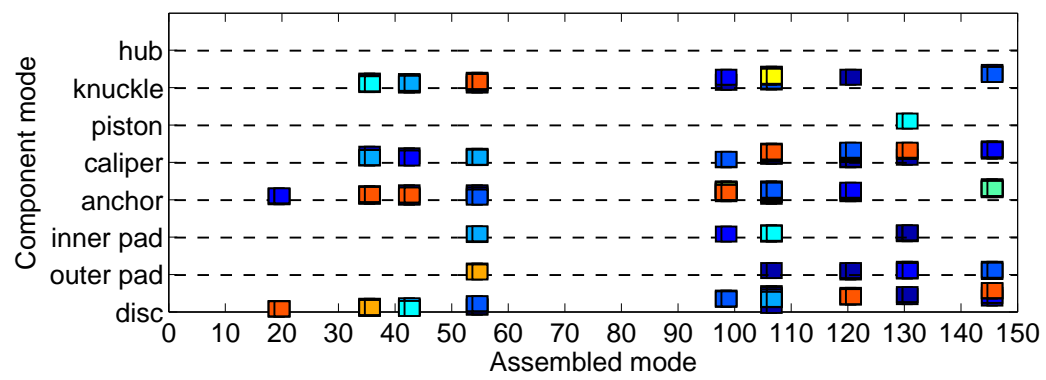

Figure 10: Sensitivity map of the first 150 system modes to all component modes. The larger the marker, the hotter the color the higher the sensitivity

The most unstable system mode (\#55, shown in figure 11) is identified as inducing squeal and must therefore be altered. A zoom in on its sensitivity study is shown in figure 12 One sees that 3 component modes are of main interest, being the ninth knuckle mode, first outer pad mode and second inner pad mode. A modification of the first outer pad mode could then be considered to stabilize the squealing mode.

$4081 \mathrm{~Hz}-2.28 \%$

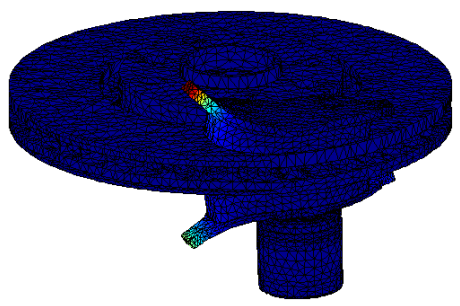

$4056 \mathrm{~Hz}-2.32 \%$

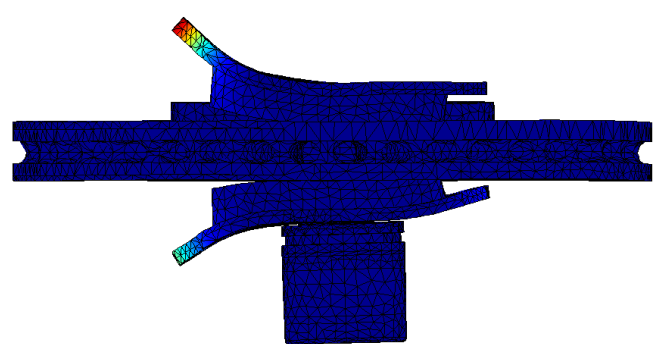

Figure 11: Brake squealing mode, partial restitution plot

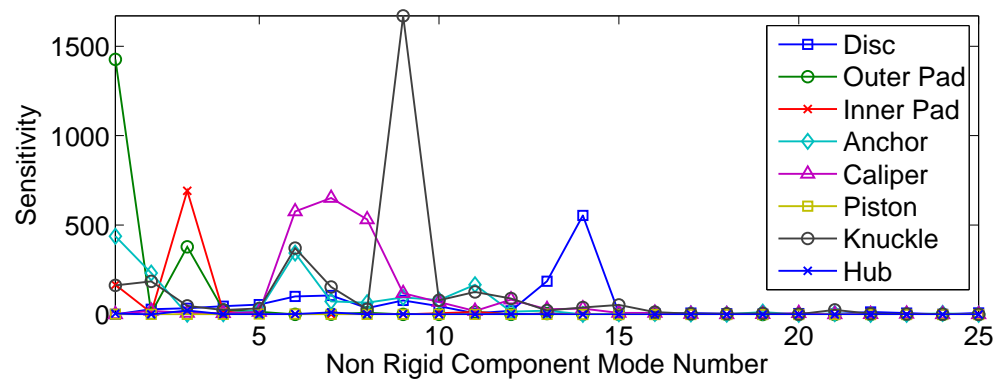

Figure 12: Mode \#55 damping sensitivity to all component modes

\section{CONCLUSION}

This paper introduced the disjoint component mode synthesis method and showed how this approach gives a clear mechanism to evaluate the impact of component modes on the system response. This evaluation is done using very compact models and can thus be used in a for systematic sensitivity studies that are needed in a design phase. 


\section{REFERENCES}

[1] Craig, R. J., A Review of Time-Domain and Frequency Domain Component Mode Synthesis Methods, Int. J. Anal. and Exp. Modal Analysis, Vol. 2, No. 2, pp. 59-72, 1987.

[2] de Klerk, D., Rixen, D. and Voormeeren, S. N., General Framework for Dynamic Substructuring : History, Review and Classification of Techniques, AIAA Journal, Vol. 46, No. 5, pp. 1169-1181, 2008.

[3] Balmes, E., Use of generalized interface degrees of freedom in component mode synthesis, International Modal Analysis Conference, pp. 204-210, 1996.

[4] Corus, M., Amélioration des méthodes de modification structurale par utilisation de techniques d'expansion et de réduction de modèle, Ph.D. thesis, École Centrale Paris, 2003.

[5] Roy, N., Abbadi, Z. and Balmes, E., Damping Specification of Automotive Structural Components via Modal Projection, PDF, September 2008.

[6] Bennighof, J., Kaplan, M., Muller, M. and Kim, M., Meeting the NVH Computational Challenge: Automated Multi-Level Substructuring, International Modal Analysis Conference, pp. 909-915, 2000.

[7] Gao, W., Li, X., Yang, C. and Bai, Z., An Implementation and Evaluation of the AMLS Method for Sparse Eigenvalue Problems, ACM Transactions on Mathematical Software, Vol. V, pp. 1-27, September 2007.

[8] Sternchüss, A., Multi-level parametric reduced models of rotating bladed disk assemblies, Ph.D. thesis, Ecole Centrale de Paris, 2009.

[9] Balmes, E., Bianchi, J. and Leclère, J., Structural Dynamics Toolbox 6.2 (for use with MATLAB), SDTools, Paris, France, www.sdtools.com, Sep 2009.

[10] Vermot des Roches, G., Balmes, E., Pasquet, T. and Lemaire, R., Time simulation of squeal phenomena in realistic brake models, Proceedings of the International Conference on Advanced Acoustics and Vibration Engineering (ISMA), pp. 3007-3019, 2008. 